\title{
1 Responses of global waterbird populations to climate change vary with latitude
}

2 Tatsuya Amano ${ }^{1,2,3^{*}}$, Tamás Székely ${ }^{4,5}$, Hannah S. Wauchope ${ }^{2}$, Brody Sandel ${ }^{6}$, Szabolcs

$3 \mathrm{Nagy}^{7}$, Taej Mundkur ${ }^{7}$, Tom Langendoen ${ }^{7}$, Daniel Blanco ${ }^{8}$, Nicole L. Michel ${ }^{9}$, William J.

4 Sutherland $d^{2,10}$

5

$6{ }^{1}$ School of Biological Sciences, University of Queensland, Brisbane, 4072 Queensland,

$7 \quad$ Australia

$8{ }^{2}$ Conservation Science Group, Department of Zoology, University of Cambridge, The David

$9 \quad$ Attenborough Building, Pembroke Street, Cambridge, CB2 3QZ, UK.

$10{ }^{3}$ Centre for the Study of Existential Risk, University of Cambridge, 16 Mill Lane, Cambridge,

11 CB2 1SG, UK.

$12{ }^{4}$ Milner Centre for Evolution, Department of Biology and Biochemistry, University of Bath,

13 Bath BA2 7AY, UK.

$14{ }^{5}$ Department of Evolutionary Zoology, University of Debrecen, Debrecen, H-4010, Hungary.

$15{ }^{6}$ Department of Biology, Santa Clara University. 500 El Camino Real, Santa Clara California, 1695053 , USA.

$17{ }^{7}$ Wetlands International Global Office, Horapark 9, 6717 LZ Ede, The Netherlands.

$18{ }^{8}$ Wetlands International Argentina, 25 de Mayo 75810 I, Buenos Aires 1002, Argentina.

$19{ }^{9}$ National Audubon Society, Conservation Science, 220 Montgomery St., Suite 1000, San

20 Francisco, CA 94104, USA.

$21{ }^{10}$ BioRISC, St Catharine's College, Cambridge CB2 1RL, UK 


\section{Abstract}

23 While climate change continues to present a major threat to global biodiversity and

24 ecosystems, most research on climate change impacts do not have the resolution to detect

25 changes in species abundance and are often limited to temperate ecosystems. This limits our

26 understanding of global responses in species abundance - a determinant of ecosystem

27 function and services - to climate change including in the highly-biodiverse tropics. We

28 address this knowledge gap by quantifying abundance responses to climate change in

29 waterbirds, an indicator taxon of wetland biodiversity, at 6,822 sites between $-55^{\circ}$ and $64^{\circ}$.

30 Using 1,303,651 count records since 1990 of 390 species, we show that with temperature

31 increase, the abundance of species and populations decreased at lower latitudes, particularly

32 in the tropics, but increased at higher latitudes. These contrasting responses to temperature

33 increase according to latitude indicate potential global-scale poleward shifts of species

34 abundance under climate change, providing empirical support for predictions by earlier

35 studies. The negative responses to temperature increase in tropical species and populations

36 are of conservation concern, as they are often also threatened by other anthropogenic factors.

37 Our results suggest that existing biases in studies towards temperate regions could

underestimate the impact of climate change on waterbirds and other species.

\section{Introduction}

41 Climate change continues to pose various serious threats to biodiversity, and there is an

42 urgent need to understand how species respond to changing climates globally. A wide range

43 of species have already shown responses to climate change, such as changes in geographical 
44 range $^{1}$, phenology ${ }^{2}$ and abundance ${ }^{3}$. However, the rate and direction of these responses vary

45 greatly among species and locations ${ }^{1,2,4}$. As climate-driven changes in biodiversity are

46 expected to affect ecosystem functioning, human well-being, and the dynamics of climate

47 change itself ${ }^{5}$, understanding how species' responses to climate change may vary globally

48 could provide crucial evidence for a more effective allocation of limited resources on a

49 priority basis for the conservation of species and ecosystems threatened the most by climate

50 change, and for assessing how climate-driven changes in biodiversity may affect human

51 societies.

52 Existing gaps in the geographical coverage of available evidence seriously limit our

53 understanding of species' responses to climate change and its variations across the globe ${ }^{6}$.

54 Earlier global reviews of species' responses to climate change have rarely incorporated

55 species and studies in the tropics, due to the lack of relevant information ${ }^{7}$. Such geographical

56 biases are even more prominent in studies investigating responses in species abundance ${ }^{8,9}$,

57 which is a major determinant of species extinction risk $^{10}$, ecosystem function and services ${ }^{11}$.

58 Research on abundance responses to climate change to date has largely been conducted in

59 Europe, North America and the Arctic ${ }^{3,12-14}$, with a recent global study showing a link

60 between climate warming and abundance declines in birds and mammals ${ }^{8}$. As a result,

61 although tropical species are predicted to be more vulnerable to increasing temperature ${ }^{15}$,

62 there is still little empirical evidence on how responses in species abundance to climate

63 change vary among and within species at the global scale.

64 Here we address this challenge by modelling global time-series data of waterbird species

65 to estimate their abundance responses to changes in temperature and precipitation. The global 
66 dataset of waterbird abundance changes used is based on long-term surveys in over 100

67 countries and covers regions for which there is little information on climate change impacts,

68 such as the tropics ${ }^{16}$. Waterbirds can also serve as an indicator taxon for assessing the status

69 of biodiversity in wetland ecosystems, which have been lost at higher rates than other

70 ecosystems, despite their high levels of biodiversity and productivity as well as the crucial

71 ecosystem functions and services delivered ${ }^{17}$.

73 sites between $-55^{\circ}$ and $64^{\circ}$ (Figure S1) we first estimated, for each species at each site, (i) the

74 rate of abundance changes with increasing temperature and precipitation as regression

75 coefficients (responses to temperature and precipitation increases), and (ii) the proportion of

76 abundance changes that can be explained independently by temperature and precipitation

77 changes (measured as $R^{2}$ ), estimated with hierarchical partitioning ${ }^{18}$ (the importance of

78 temperature and precipitation). We then tested multiple hypotheses that are rarely explored at

79 the global scale (Supplementary Tables S1 and S2), to examine among- and within-species

80 variations in responses to temperature and precipitation increases as well as the importance of

81 temperature and precipitation across latitudes.

\section{Results}

83 Applying the Gompertz model of population growth to the global waterbird dataset enabled

84 us to estimate abundance responses to the changes in temperature and precipitation at $1^{\circ} \times 1^{\circ}$

85 grid cells across latitudes, including the tropics, for a wide range of waterbird groups. Of the

86390 species analysed, 144 species (36.9\%) had at least one estimate in the tropics and 129 
87 species $(33.1 \%)$ had their absolute latitudinal range mid-points in the tropics (defined as

88 tropical species; Figure 1).

89 Many species showed considerable spatial variation in abundance responses to

90 temperature increases within their geographical ranges, with particularly negative responses

91 in the tropics (Figure 1), although the importance of temperature in explaining abundance

92 changes tended to be low across the ranges, with an overall median $R^{2}$ of 0.057

93 (Supplementary Data S1 and S2). In contrast, for most species there was no clear

94 geographical pattern in abundance responses to precipitation increases, and precipitation was

95 found to have a low importance in explaining abundance changes (the overall median $R^{2}=$

96 0.051; Supplementary Data S1 and S2). These geographical patterns were also evident in the

97 distribution of abundance responses averaged across all species observed within each grid

98 cell; species generally showed more negative responses to temperature increases at lower

99 latitudes, such as in South and Southeast Asia (Figure 2).

For 213 species with estimates at ten or more grid cells, we then tested hypotheses on

how responses to temperature and precipitation increases (the rate of abundance changes with increasing temperature/precipitation) and the importance of temperature and precipitation

(the proportion of abundance changes that can be explained by temperature/precipitation changes) vary among species (among each species' estimates at latitudinal range mid-points; 
109

110

111

112

113

S2a). When compared within species, although 198 (93\%) out of the 213 species showed more negative responses to temperature increases at lower latitudes, this within-species latitudinal pattern was significant only in eight of the 198 species (Figure 3b, Supplementary Data S3). The importance of temperature in explaining abundance changes also increased with latitude among species (Figure 3c, S2b) and within species for all 55 species with a significant within-species latitudinal pattern (Figure 3d, Supplementary Data S3). Migratory species, larger-sized species and species with a wider latitudinal range showed a higher importance of temperature in explaining abundance changes (Figure S2b).

In contrast, neither abundance responses to precipitation increases nor the importance of precipitation in explaining abundance changes showed significant latitudinal patterns among species, although, for some species in the tropics, precipitation was found to have a relatively high importance in explaining abundance changes (Figure 4a, c, S3 and Supplementary Data S3). Precipitation was shown to have a higher importance in explaining abundance changes in species with a wider latitudinal range (Figure S3b). When compared within species, three species showed a significant, one species showed a decrease and another species showed a hump-shaped curve in abundance responses to precipitation increases along latitudes (Figure 4b, Supplementary Data S3). The importance of precipitation in explaining abundance changes showed a significant within-species latitudinal pattern for just one species (Figure 4d, Supplementary Data S3).

\section{Discussion}

Our results demonstrate the responses in waterbird abundance to temperature increases differ between tropical and non-tropical regions. At both species and population levels, waterbird 
131

132

abundance generally decreased in the tropics, but increased at higher latitudes, with increasing temperature. This supports our predictions on among- and within-species patterns (Supplementary Table S1). Species in the tropics tend to live closer to their upper temperature limits ${ }^{15}$, have a narrower temperature niche ${ }^{19}$ and change their temperature niche at a slower rate ${ }^{20}$, all of which indicate that tropical species are more vulnerable to increasing temperatures at the species level. Climate-related extinctions of local populations, typically at the warmer edge of the species' geographical range, are also more frequent in the tropics, causing poleward range shifts in many species ${ }^{21}$. While such species-level and populationlevel responses to climate change have often been investigated separately to date, our results provide novel empirical evidence that impacts of temperature increases on tropical ecosystems can be characterised by species-wide declines in tropical species as well as population-level responses in wider-ranging species.

Nevertheless, we also found that temperature generally explains only a small proportion of yearly abundance changes in waterbirds, especially in tropical species and at the lowlatitude range margin of species, possibly for four reasons. First, the effect of temperature changes on waterbird abundance can be indirect especially at lower latitudes. Although warmer weather conditions can directly increase the survival of waterbirds at higher latitudes $^{22}$, indirect biotic processes (e.g., changes in food availability), rather than direct abiotic processes (e.g., heat stress), are reported to be more important mechanisms for climate-driven abundance changes, especially for higher-level consumers like birds ${ }^{23,24}$. For example, increases in already-high temperatures at lower latitudes can cause wetlands to dry, reducing the availability of habitats and food resources for waterbirds ${ }^{22}$. Such an indirect 
153 effect of temperature increases could have obscured the relationship between changes in

154 temperature and abundance, especially in the tropics and at the low-latitude range margins.

155 Second, many of the waterbirds analysed here are migratory species, which generally have a

156 higher dispersal ability ${ }^{25}$ and track climate niches to a greater extent than resident species ${ }^{26}$,

157 and thus can be more responsive to changes in local temperature; this was supported by the

158 positive effect of migratory status on the importance of temperature (Figure S2b). In this

159 study more non-tropical species tended to be migratory compared to tropical species (151

160 (96\%) of 158 non-tropical species and 12 (78\%) of 55 tropical species were migratory),

161 which may explain the higher importance of local temperature in explaining the abundance of

162 non-tropical species. Third, observation errors can cause a lower explanatory power of

163 variables. We may expect larger errors in the tropics, compared to temperate regions, where

164 waterbird surveys have a longer history and thus surveyors might be better trained. Finally,

165 other important threats, such as habitat loss and hunting, affect bird abundance,

166 independently from, or synergistically with, climate change ${ }^{27}$. By testing the effect of

167 temperature and precipitation changes on yearly abundance changes while estimating long-

168 term growth rates, our modelling approach controlled for the consistent impacts of such

169 threats on long-term trends in abundance (see Statistical Analyses for more detail).

170 Nevertheless, those threats can also cause yearly abundance changes and their impacts are

171 likely to be more severe at lower latitudes ${ }^{16}$, potentially causing temperature to have lower

172 importance at lower latitudes.

173 Contrary to our hypotheses, there was no clear latitudinal pattern in abundance responses

174 to precipitation changes, either among or within species. In general water availability, 
175 compared to ambient temperature, has been shown to be a more important driver of species

176 richness and population size at lower latitudes ${ }^{4,28}$. Supporting this, our results showed that

177 precipitation was more important in explaining the abundance of some tropical species

178 compared to most species in higher latitudes, although the overall among-species pattern

179 across latitudes was not significant (Figure 4c). This may be explained by the following two

180 reasons. First, precipitation changes can affect waterbird species at the river basin scale (often

181 the scale of 500 to $1,000 \mathrm{~km}$ ) through effects on water flow into their wetland habitats ${ }^{29,30}$.

182 Therefore, our analysis at the resolution of $1^{\circ}$ grid cells (equivalent to a grain size of 96.49

$183 \mathrm{~km}$ ) may not have been able to detect such a broad-scale impact of precipitation changes.

184 Second, waterbird responses to precipitation changes can vary greatly among species, as we

185 recognised when developing our hypotheses (Supplementary Table S1). While increased

186 rainfall generally leads to more favourable habitat conditions for waterbirds in dry regions ${ }^{22,31}$,

187 elevated water levels associated with increased rainfall can cause the loss of shallow-water

188 habitats, often followed by abundance decreases in certain groups, such as shorebirds ${ }^{32}$. Such

189 mixed responses to precipitation changes among species ${ }^{33}$ may have resulted in the lack of

190 clear latitudinal patterns, particularly among species, in this study.

191 Our results point to three major implications on the impact of climate change on global

192 biodiversity. First, despite the relatively low importance of temperature in explaining

193 waterbird abundance changes, all other things being equal, local temperature increases under

194 ongoing climate change are likely to pose a more negative impact on species and populations

195 in the tropics. This provides important evidence for the climate-driven degradation of tropical

196 ecosystems, which has recently been under debate ${ }^{34,35}$. Although climate change is not the 
197 only threat to waterbird species, impacts of other major threats, such as loss and degradation

198 of wetlands and excessive hunting pressure, seem to be more severe in the tropics too ${ }^{16}$,

199 indicating that tropical species and populations suffer from multiple anthropogenic threats.

200 Second, the revealed negative impact of temperature increases in the tropics suggests that

201 existing severe biases in scientific studies and data towards temperate regions could

202 underestimate the impact of climate change on species populations at the global scale.

203 Highlighting the negative impact of climate change on tropical waterbirds should serve to

204 inspire further studies on other taxa in the tropics. Finally, our finding of contrasting

205 abundance responses to temperature increases according to latitude highlights the possibility

206 of global-scale poleward shifts in abundance across species, and associated ecosystem

207 functions and services. As such shifts can have serious consequences not only for biodiversity

208 but also for human well-being, assessing latitudinal patterns in biodiversity responses to

209 climate change at the population, species and community levels warrants further research

210 attention. 
Methods

\section{Data}

213 Waterbird count data

214 Data used in this study comprised site-specific annual counts based on the International

215 Waterbird Census (IWC) coordinated by Wetlands International and the Christmas Bird

216 Count (CBC) by the National Audubon Society in the USA, and were compiled in our earlier

217 study $^{16}$. Note that counts based on these surveys should be described as relative abundance,

218 as we could not account for imperfect detections in this study. However, we have referred to

219 them as abundance throughout the manuscript for simplicity. Nevertheless, these count

220 records should still be comparable among years (see section Model for estimating abundance

221 responses for more detail).

222 The IWC, launched in 1967, is a scheme for monitoring waterbird numbers, covering

223 more than 25,000 sites in over 100 countries with more than 15,000 observers. The

224 coordination of the IWC is divided into four regional schemes corresponding to the major

225 migratory flyways of the world: the African-Eurasian Waterbird Census (AEWC), Asian

226 Waterbird Census (AWC), Caribbean Waterbird Census (CWC) and Neotropical Waterbird

227 Census (NWC). We did not use data from the CWC, as, having started in 2010, it only

228 provides short-term data. The survey methodology is essentially the same across the four

229 regional schemes. Population counts are typically carried out once every year in mid-January

230 but may include counts between December to February. Additional counts are also conducted

231 in other months, particularly in July in the Southern Hemisphere, but we only used the

232 January and February counts for consistency. This means that our data from the Northern

233 Hemisphere are for non-breeding populations while those in the Southern Hemisphere also

234 include some breeding populations. In each country that is covered by the survey, national 
coordinators manage an inventory of wetland sites (hereafter, survey sites), including sites of

236 international- or national-level recognition (e.g., Ramsar sites, Flyway Network Sites,

237 Important Bird Areas, national parks etc.). Each survey site is generally defined by

238 boundaries so that observers know precisely which areas are to be covered in the surveys.

239 The observers consist of a wide variety of volunteers, but national coordinators usually train

240 them using materials produced by Wetlands International to ensure the quality of count data.

241 Survey sites (normally up to a few $\mathrm{km}^{2}$ ) are typically surveyed by about two observers for up

242 to four hours, while larger sites can require a group of observers working over several days.

243 Most surveys are conducted on foot, or from a vehicle, with boats involved in a few. The time

244 of survey on any given day depends on the type of survey site: inland sites are normally

245 surveyed during the morning or late afternoon, whereas coastal sites are surveyed over the

246 high tide period (mangrove areas and nearby mudflats are, however, covered during low

247 tides). Surveys cover waterbirds, defined as bird species that are ecologically dependent on

248 wetlands ${ }^{36}$. Counts are usually made by scanning flocks of waterbirds with a telescope or

249 binoculars and counting each species. Zero counts are not always recorded, and thus are

250 inferred using a set of criteria (see below for more detail). Count records, together with

251 associated information, are submitted to the national coordinators, who compile the submitted

252 records, check their validity and submit those records to Wetlands International. See ${ }^{36,37}$ for

253 more details on survey methodology.

254 As the IWC does not cover North America, we also used data based on the CBC, which

255 has been conducted annually since 1900, and now includes over 2,400 count circles (defined

256 as survey sites in this study) and involves more than 70,000 observers each year ${ }^{38}$. Each CBC

257 consists of a tally of all bird species detected within $24.1 \mathrm{~km}$ in diameter, on a single day

258 between 14th December and 5th January. The majority of circles (and most historical data) 
are from the US and Canada. Observers join groups that survey subunits of the circle during the course of the day using a variety of transportation methods (mostly on foot, or in a car, but can include boats, skis, or snowmobiles). The number of observers and the duration of counts vary among circles and through time. The total number of survey hours per count has been recorded as a covariate to account for the variable duration of and participation in the count. We only used records on waterbird species in this paper.

We compiled data from each scheme by species, except for data based on the AfricanEurasian Waterbird Census, where data had already been stored by flyway for each species ${ }^{37}$. As data based on the Neotropical Waterbird Census are only available for 1990 onward, we only used post-1990 data for other regions as well. The latest records were in 2013. For the IWC data, we generated zero counts using an established approach ${ }^{37}$, in which we started with a list of all species observed in each country and assumed a zero count of any species that were on the list but not recorded at a particular site on a particular day if the site was surveyed on that day, as shown by the presence of any other species' record(s), and if no multi-species code related to the species (e.g., Anatinae spp. for species of the genus Anas) was recorded for the site-date combination. We projected all survey sites onto a Behrmann equal-area cylindrical projection and assigned them to grid cells with a grain size of $96.49 \mathrm{~km}$, or approximately $1^{\circ}$ at $30^{\circ} \mathrm{N} / \mathrm{S}$. We only used species that were observed at one or more survey sites for ten or more years since 1990, and this has resulted in 390 species being analysed in this study (see Supplementary Data S4 for the full list of species). Species groups used in Fig. 1 are based on the International Ornithological Congress World Bird List ${ }^{39}$ : coursers, gulls, terns and auks (Alcidae, Glareolidae, Laridae and Stercorariidae), grebes and flamingos (Phoenicopteridae and Podicipedidae), loons and petrels (Gaviidae and Procellariidae), pelicans, boobies and cormorants (Anhingidae, Fregatidae, Pelecanidae, 
283 Phalacrocoracidae and Sulidae), rails and cranes (Aramidae, Gruidae and Rallidae),

284 shorebirds (Burhinidae, Charadriidae, Haematopodidae, Jacanidae, Recurvirostridae,

285 Rostratulidae and Scolopacidae), storks, ibises and herons (Ardeidae, Ciconiidae and

286 Threskiornithidae), and waterfowl (Anatidae and Anhimidae).

287

\section{Explanatory variables}

289 To estimate responses in waterbird abundance to changes in temperature and precipitation,

290 we used monthly mean temperature and precipitation total in the CRU TS v. 4.01 database ${ }^{40}$,

291 by assigning each site to the $0.5^{\circ}$ climatic grid cell including the site. When testing among-

292 and within-species latitudinal patterns in abundance responses, we also accounted for three

293 species-level variables—-latitudinal geographical range, migratory status and body size — that

294 are expected to explain among-species variations in responses: data sources of those variables

295 are shown in Supplementary Table S2.

297 Statistical Analyses

298 Model for estimating abundance responses

299 We first estimated for each species at each survey site the rate of abundance changes with

300 increasing temperature and precipitation as regression coefficients (defined as abundance

301 responses to temperature or precipitation increases) by applying the Gompertz model of

302 population growth to count records:

303

$$
N_{t}=N_{t-1} \exp \left(\alpha+\beta_{1} \log N_{t-1}+\beta_{2} \text { Temp }_{t-1}+\beta_{3} \text { Prec }_{t-1}\right),
$$

304 where $N_{t}$, Temp $_{t}, \operatorname{Prec}_{t}$ are the abundance of the species, the relevant mean Dec-Feb

305 temperature and precipitation at the site in year $t$, respectively. $\beta_{1-3}$ are regression coefficients 
and $\alpha$ is the intercept. By estimating $\alpha$ as the population growth rate, this model tests the

307

308

effect of temperature and precipitation on yearly changes in abundance while controlling for long-term trends in abundance. This model structure helps to avoid detecting a spurious relationship between long-term trends in abundance caused by other threats (e.g., long-term declines by habitat loss) and those in temperature or precipitation (e.g., long-term warming temperatures). Taking logs and rearranging to express in terms of relative growth rate result in the following form:

$$
\log N_{t}-\log N_{t-1}=\alpha+\beta_{1} \log N_{t-1}+\beta_{2} \text { Temp }_{t-1}+\beta_{3} \text { Prec }_{t-1}
$$

and we used this form to estimate regression coefficients with linear models in R 3.4.1 ${ }^{41}$. As this model does not allow missing values, any missing values between the first and last survey years at each site for each species were replaced by linear interpolation using the package $\mathrm{zoo}^{42}$; the proportion of missing values (i.e., the effect of interpolation) was accounted for in the following analysis (see Latitudinal analysis). The estimated $\beta_{2}$ and $\beta_{3}$ represent site-level abundance responses to temperature and precipitation increases, respectively. Using the same model we also estimated the site-level independent capacity of temperature and precipitation changes in explaining abundance changes (defined as the importance of temperature and precipitation) with hierarchical partitioning ${ }^{18}$ (measured in our case as $\mathrm{R}^{2}$ ) using the package hier.part ${ }^{43}$.

As the model described above tests the effect of temperature and precipitation in the previous year (i.e., year $t-1$ ) on abundance in the survey year (year $t$ ), we also separately tested the immediate effect of temperature and precipitation in the same year (year $t$ ) as the abundance survey year. For this analysis we used mean temperature and precipitation in 
328

December (year $t-1$ ), January and February (year $t$ ) for the IWC sites, where surveys were conducted either in January or February, and mean Dec temperature and precipitation in year $t$ for the CBC sites, where surveys were largely conducted in December. We compared AIC of the two models at each site for each species and used the coefficients in the model with a smaller AIC.

We assumed constant survey efforts over time for the IWC, because regular and standardized surveys with constant methods, efforts and timing are strongly encouraged in this scheme (see Supplementary Discussion in ${ }^{16}$ for more detail). However, survey efforts in the $\mathrm{CBC}$ are known to vary through time. Following a previously published analysis ${ }^{44}$ we thus accounted for the survey effort effect for the $\mathrm{CBC}$ data by using the total number of survey hours per count as the measure of survey efforts:

$$
\log N_{t}-\log N_{t-1}=\alpha+\beta_{1} \log N_{t-1}+\beta_{2} \text { Temp }_{t-1}+\beta_{3} \operatorname{Prec}_{t-1}+\frac{\mathrm{B}\left(\left(\frac{\zeta \mathrm{t}}{\bar{\zeta}}\right)^{\mathrm{p}}-1\right)}{\mathrm{p}}
$$

where $\zeta_{t}$ is the total number of survey hours per count and $\bar{\zeta}$ is the mean value of $\zeta_{t}$. The parameters $\mathrm{B}$ and $\mathrm{p}$ determine a range of relationships between effort and the number of birds counted $^{44}$ and we used the values estimated for each species in our earlier study ${ }^{16}$ (see

\section{Supplementary Data S4).}

We only used survey sites with ten or more records and five or more non-zero records since 1990 for at least one species, and this has resulted in 1,303,651 count records since 1990 on 390 species at 6,822 sites between $-55^{\circ}$ and $64^{\circ}$ (Supplementary Figure S1) being analysed in this study. We aggregated the estimated site-level responses to temperature and precipitation increases as well as the importance of temperature and precipitation to $1^{\circ} \times 1^{\circ}$ grid cells by calculating the mean site-level estimates across all sites in each grid cell, 
weighted by the inverse of estimate variance at each site to account for uncertainties. The grid

cell-level estimates (Supplementary Data S2) were then used in the latitudinal analysis species to account for uncertainties.

\section{Latitudinal analysis}

358 We used absolute latitudes to test latitudinal patterns described in Supplementary Table S1

359 for the following reason. Our data include species that are distributed only in either the northern or southern hemisphere (one-hemisphere species) as well as those that appear in both the hemispheres (two-hemisphere species). Some of our hypotheses (e.g., that for among-species patterns in abundance changes with increasing temperature, shown at the top of Supplementary Table S1) predict that one-hemisphere species would show a monotonic increase with raw latitudes while two-hemisphere species would show a U-shaped relationship along the raw latitudinal gradient with the lowest point at the equator; this makes analysing those species together in the hierarchical modelling framework described below a complicated process. With absolute latitudes, in contrast, one-hemisphere and twohemisphere species are both expected to show a monotonic increase, making the parameter estimation much simpler.

To explain among- and within-species latitudinal variations in abundance responses to temperature and precipitation changes as well as the importance of temperature and

372 precipitation for 213 species with estimates at ten or more grid cells, we adopted the within- 
373 subject centring approach ${ }^{45}$ under a hierarchical modelling framework to explicitly

374 distinguish species-level effects (explaining variations in species-level responses between

375 species) and population-level effects (explaining variations in population-level responses

376 within species) of explanatory variables. Here we defined each species responses at their

377 absolute latitudinal range mid-points as species-level responses, and responses within each

378 grid cell as population-level responses.

379 In this model the species effect $\mu_{s}$, representing the species-level responses to temperature

380 or precipitation increases in species $s$, is drawn from a normal distribution with mean of $v_{s}$

381 and variance of $\sigma_{v}^{2} \cdot v_{s}$ is further modelled with species-level explanatory variables:

$$
v_{s}=\alpha+\beta_{B 1} M I D L A T_{S}+\beta_{B 2} M I D L A T_{s}^{2}+\beta_{B 3} L A T R A N G E_{S}+\beta_{B 4} M I G_{s}+\beta_{5} B M_{B s}+
$$$$
\beta_{B 6} P R O P N A_{s}+\eta_{s}
$$

where $\alpha$ is the global intercept and $\beta_{B 1-B 6}$ represent the species-level effects. MIDLAT,

$L A T R A N G E_{s}, M I G_{s}, B M_{s}, P R O P N A_{s}$ are species-level explanatory variables; absolute

latitudinal range mid-points, absolute latitudinal geographical range (degree), migration missing values (i.e., interpolated values) in count records across all sites (\%) for species $s$, respectively. $\eta_{s}$ is a random term that accounts for phylogenetic dependence among species and is drawn from a multivariate normal distribution ${ }^{46,47}$ :

$$
\begin{aligned}
& \eta_{s} \sim \operatorname{MVN}\left(\mathbf{0}, \delta^{2} \Sigma_{\lambda}\right), \\
& \Sigma_{\lambda}=\lambda \Sigma+(1-\lambda) \mathbf{I},
\end{aligned}
$$

where $\Sigma$ is a scaled variance-covariance matrix calculated from an ultrametric phylogenetic tree (defined below). By scaling $\Sigma$ to a height of one, we can interpret $\delta^{2}$ as the residual 
into the matrix with the identity matrix $\mathbf{I}$. Here $\lambda$ is a coefficient that multiplies the off-

diagonal elements of $\Sigma$ and a $\lambda$ close to zero implies that the phylogenetic signal in the data is

low, suggesting independence in the error structure of the data points, whereas a $\lambda$ close to

one suggests a good agreement with the Brownian Motion evolution model and thus suggests correlation in the error structure ${ }^{46,49}$. To incorporate uncertainties ${ }^{50}$ in phylogenetic trees in the calculation of $\Sigma$, we used a sample of 100 trees from a comprehensive avian phylogeny ${ }^{51}$ as the prior distribution for our analysis ${ }^{46}$. More specifically, one of the 100 trees was randomly drawn in each iteration and used for the calculation of $\Sigma$. grid cell $i$ was then assumed to derive from a normal distribution with mean $\mu_{s, i}$ and variance $\sigma_{\mu}^{2}$, where $\mu_{s, i}$ is modelled using the species effect $\mu_{s}$ :

Here $\beta_{W s, 1-3}$ represents the population-level effect of absolute latitudes $L A T_{s, i}$ (in the form of values (i.e., interpolated values) in count records across all sites $p N A_{s, i}(\%)$ of grid cell $i$ for species $s$. Here within-species variations in population-level responses $\left(\mu_{s, i}-\mu_{s}\right)$ are explained by within-species variations in absolute latitudes $\left(L A T_{s, i}-M I D L A T_{s}\right)$, divided by

415 The species-specific $\beta_{W s, 1-3}$ is the random effect each governed by hyper-parameters as:

$$
\beta_{W_{s, j}} \sim \operatorname{Normal}\left(h \beta_{W_{j}}, \sigma_{\beta_{W_{j}}}^{2}\right)
$$

$417 \gamma_{s, i}$ accounts for spatial autocorrelation within each species and is drawn from an intrinsic

418 Gaussian conditional autoregressive (CAR) prior distribution with variance $\sigma_{\gamma_{s}}^{2}$ : 


$$
\gamma_{s, i} \mid \gamma_{s, k} \sim \operatorname{Normal}\left(\frac{\sum_{i \neq k} w_{i, k} \gamma_{s, k}}{n_{i}}, \frac{\sigma_{\gamma s}^{2}}{n_{i}}\right)
$$

420 where $w_{i, k}=1$ if grid cells $i$ and $k$ are neighbours, and 0 otherwise. $n_{i}$ is the total number of

neighbours of grid cell $i$ and neighbours here are defined as those grid cells directly adjacent,

422 including those diagonal. $\sigma_{\gamma s}{ }^{2}$ controls the amount of variation between the random effects.

We tested latitudinal patterns in the importance of temperature and precipitation using

with mean $c_{s, i}$ and variance $\frac{\alpha_{i} \beta_{i}}{\left(\alpha_{i}+\beta_{i}\right)^{2}\left(\alpha_{i}+\beta_{i}+1\right)}$ with a logit link function:

$$
\operatorname{logit}\left(c_{s, i}\right)=\mu_{s}+\beta_{w_{s, 1}} \frac{L A T_{s, i}-M I D L A T_{s}}{L A T R A N G E_{s}}+\beta_{w_{s, 2}}\left(\frac{L A T_{s, i}-M I D L A T_{s}}{L A T R A N G E_{S}}\right)^{2}+\beta_{w_{s, 3}} p N A_{s . i}+\gamma_{s, i}
$$

The models were implemented with OpenBUGS $3.2 .3^{52}$ and the R2OpenBUGS package ${ }^{53}$

in $\mathrm{R}$ 3.4.1 $1^{41}$. As non-informative prior distributions, we used a Gamma distribution with mean of 1 and variance of 100 for $\varphi_{i}$ and the inverse of $\sigma_{v}^{2}, \delta^{2}, \sigma_{\mu}^{2},{\sigma_{\beta_{W_{j}}}^{2}}^{2}$ and $\sigma_{\gamma_{s}}^{2}$, a uniform

distribution on the interval $[0,1]$ for $\lambda$, normal distributions with mean of 0 and variance of 100 for $\alpha, \beta_{B k}$, and $h \beta_{W j}$. Each MCMC algorithm was run with three chains with different initial values for 30,000 iterations with the first 10,000 discarded as burn-in and the remainder thinned to one in every four iterations to save storage space. Model convergence was checked with R-hat values. 
442 herring gull Larus argentatus and Arctic herring gull L. smithsonianus, and common

443 moorhen Gallinula chloropus and common gallinule G. galeata. Larus glaucoides thayeri

444 was excluded from the latitudinal analysis as it is not included in either database. We also

445 excluded from the analysis eight seabird species in Alcidae and Sulidae as neither the IWC

446 nor CBC necessarily targets seabird species.

447 We also used R packages ape ${ }^{55}$, data.table ${ }^{56}$, dplyr $^{57}$, ggplot $^{58}{ }^{\text {gridExtra }}{ }^{59}$, mapdata $^{60}$,

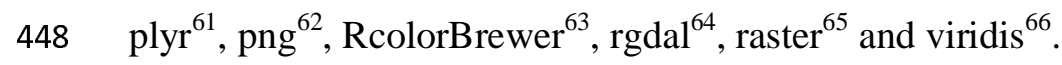

450 Data Availability

451 The waterbird count data used in this study are collated and managed by Wetlands

452 International and the National Audubon Society, and are available from Wetlands

453 International at: http://iwc.wetlands.org/. The estimated abundance responses to temperature

454 and precipitation as well as the importance of temperature and precipitation for each grid cell

455 for each species are available as Supplementary Data S2. All the data on explanatory

456 variables are freely available as specified in Supplementary Table S2.

\section{Code Availability}

459 All the R codes used for the analyses are available as Supplementary Data S5-7. 


\section{References}

4621 Chen, I. C., Hill, J. K., Ohlemüller, R., Roy, D. B. \& Thomas, C. D. Rapid range shifts of

463 species associated with high levels of climate warming. Science 333, 1024-1026 (2011).

4642 Thackeray, S. J. et al. Phenological sensitivity to climate across taxa and trophic levels.

$465 \quad$ Nature 535, 241 (2016).

4663 Stephens, P. A. et al. Consistent response of bird populations to climate change on two

467 continents. Science 352, 84-87 (2016).

4684 Pearce-Higgins, J. W. et al. Geographical variation in species' population responses to

469 changes in temperature and precipitation. Proceedings of the Royal Society of London B:

$470 \quad$ Biological Sciences 282, 20151561 (2015).

4715 Pecl, G. T. et al. Biodiversity redistribution under climate change: Impacts on ecosystems

$472 \quad$ and human well-being. Science 355, eaai9214 (2017).

4736 Perez, T. M., Stroud, J. T. \& Feeley, K. J. Thermal trouble in the tropics. Science 351,

$474 \quad 1392-1393(2016)$.

4757 Feeley, K. J., Stroud, J. T., Perez, T. M. \& Kühn, I. Most 'global' reviews of species'

476 responses to climate change are not truly global. Diversity and Distributions 23, 231-234

$477 \quad$ (2017).

4788 Spooner, F. E. B., Pearson, R. G. \& Freeman, R. Rapid warming is associated with

479 population decline among terrestrial birds and mammals globally. Global Change

$480 \quad$ Biology 24, 4521-4531 (2018).

4819 Martay, B. et al. Impacts of climate change on national biodiversity population trends.

$482 \quad$ Ecography 40, 1139-1151 (2017).

10 IUCN. IUCN Red List Categories and Criteria: Version 3.1. (Species Survival

484 Commission. IUCN, 2001). 
48511 Winfree, R., W. Fox, J., Williams, N. M., Reilly, J. R. \& Cariveau, D. P. Abundance of

486 common species, not species richness, drives delivery of a real-world ecosystem service.

$487 \quad$ Ecology Letters 18, 626-635 (2015).

48812 Mills, S. C. et al. European butterfly populations vary in sensitivity to weather across 489 their geographical ranges. Global Ecology and Biogeography 26, 1374-1385 (2017).

49013 Bowler, D. E. et al. Cross-realm assessment of climate change impacts on species'

$491 \quad$ abundance trends. Nature Ecology \& Evolution 1, 0067 (2017).

49214 Myers-Smith, I. H. et al. Climate sensitivity of shrub growth across the tundra biome.

$493 \quad$ Nature Climate Change 5, 887-892 (2015).

49415 Khaliq, I., Hof, C., Prinzinger, R., Böhning-Gaese, K. \& Pfenninger, M. Global variation 495 in thermal tolerances and vulnerability of endotherms to climate change. Proceedings of 496 the Royal Society B: Biological Sciences 281, 20141097 (2014).

49716 Amano, T. et al. Successful conservation of global waterbird populations depends on 498 effective governance. Nature 553, 199-202 (2018).

499

500

501

502

503

504

505

506

507

17 Ramsar Convention on Wetlands. Global Wetland Outlook: State of the World's Wetlands and their Services to People. (Ramsar Convention Secretariat, 2018).

18 Mac Nally, R. Hierarchical partitioning as an interpretative tool in multivariate inference. Australian Journal of Ecology 21, 224-228 (1996).

19 Cadena, C. D. et al. Latitude, elevational climatic zonation and speciation in New World vertebrates. Proceedings of the Royal Society B: Biological Sciences 279, 194-201 (2012).

20 Jezkova, T. \& Wiens, J. J. Rates of change in climatic niches in plant and animal populations are much slower than projected climate change. Proceedings of the Royal Society B: Biological Sciences 283, 20162104 (2016). 
50821 Wiens, J. J. Climate-related local extinctions are already widespread among plant and

$509 \quad$ animal species. PLOS Biology 14, e2001104 (2016).

51022 Maclean, I. M. D., Rehfisch, M. M., Delany, S. \& Robinson, R. A. The effects of climate

511 change on migratory waterbirds within the African-Eurasian flyway. (The Agreement on

512 the Conservation of African-Eurasian Migratory Waterbirds Secretariat, 2007).

51323 Ockendon, N. et al. Mechanisms underpinning climatic impacts on natural populations:

514 altered species interactions are more important than direct effects. Global Change

515 Biology 20, 2221-2229 (2014).

51624 Cahill, A. E. et al. How does climate change cause extinction? Proc. R. Soc. B-Biol. Sci.

$517 \quad$ 280, doi:20121890 (2013).

518

25 Angert, A. L. et al. Do species' traits predict recent shifts at expanding range edges? Ecology Letters 14, 677-689 (2011).

26 Gómez, C., Tenorio, E. A., Montoya, P. \& Cadena, C. D. Niche-tracking migrants and niche-switching residents: evolution of climatic niches in New World warblers (Parulidae). Proceedings of the Royal Society B: Biological Sciences 283, 20152458 (2016).

27 Betts, M. G., Gutiérrez Illán, J., Yang, Z., Shirley, S. M. \& Thomas, C. D. Synergistic effects of climate and land-cover change on long-term bird population trends of the western USA: a test of modeled predictions. Frontiers in Ecology and Evolution 7 (2019).

28 Hawkins, B. A. et al. Energy, water, and broad-scale geographic patterns of species richness. Ecology 84, 3105-3117 (2003).

29 Haig, S. M., Murphy, S. P., Matthews, J. H., Arismendi, I. \& Safeeq, M. Climate-altered wetlands challenge waterbird use and migratory connectivity in arid landscapes. Scientific Reports 9, 4666 (2019). 
53230 Kingsford, R. T., Bino, G. \& Porter, J. L. Continental impacts of water development on

533 waterbirds, contrasting two Australian river basins: Global implications for sustainable

$534 \quad$ water use. Global Change Biology 23, 4958-4969 (2017).

53531 Kleijn, D., Cherkaoui, I., Goedhart, P. W., van der Hout, J. \& Lammertsma, D.

536 Waterbirds increase more rapidly in Ramsar-designated wetlands than in unprotected

537 wetlands. Journal of Applied Ecology 51, 289-298 (2014).

53832 Canepuccia, A. D., Isacch, J. P., Gagliardini, D. A., Escalante, A. H. \& Iribarne, O. O.

539 Waterbird response to changes in habitat area and diversity generated by rainfall in a SW

$540 \quad$ Atlantic coastal lagoon. Waterbirds 30, 541-553 (2007).

54133 Romano, M., Barberis, I., Pagano, F. \& Maidagan, J. Seasonal and interannual variation

542 in waterbird abundance and species composition in the Melincu テウ saline lake, Argentina.

$543 \quad$ European Journal of Wildlife Research 51, 1-13 (2005).

54434 Lister, B. C. \& Garcia, A. Climate-driven declines in arthropod abundance restructure a

$545 \quad$ rainforest food web. Proceedings of the National Academy of Sciences 115, E10397-

$546 \quad$ E10406 (2018).

54735 Willig, M. R. et al. Populations are not declining and food webs are not collapsing at the

548 Luquillo Experimental Forest. Proceedings of the National Academy of Sciences 116,

$549 \quad$ 12143-12144 (2019).

55036 Delany, S. Guidance on waterbird monitoring methodology: field protocol for waterbird

$551 \quad$ counting. (Wetlands International, 2010).

55237 van Roomen, M., van Winden, E. \& van Turnhout, C. Analyzing population trends at the

553 flyway level for bird populations covered by the African Eurasian Waterbird Agreement:

$554 \quad$ details of a methodology. (SOVON Dutch Centre for Field Ornithology, 2011).

55538 LeBaron, G. S. The 115th Christmas Bird Count. (National Audubon Society, 2015). 
55639 Gill, F. \& Donsker, D. IOC World Bird List (v 5.1) (Available at:

557 http://www.worldbirdnames.org/, 2015).

55840 Harris, I., Jones, P. D., Osborn, T. J. \& Lister, D. H. Updated high-resolution grids of

559 monthly climatic observations - the CRU TS3.10 Dataset. International Journal of

$560 \quad$ Climatology 34, 623-642 (2014).

561

562

563

564

565

566

567

568

569

570

571

572

573

574

575

576

577

578

579

$41 \mathrm{R}$ Core Team. R: A language and environment for statistical computing. (R Foundation for Statistical Computing, 2017).

42 Zeileis, A. \& Grothendieck, G. zoo: S3 infrastructure for regular and irregular time series. Journal of Statistical Software 14, 1-27 (2005).

43 Walsh, C. \& Nally, R. M. hier.part: hierarchical partitioning. $R$ package version 1.0-4. (2013).

44 Link, W. A. \& Sauer, J. R. Seasonal components of avian population change: joint analysis of two large-scale monitoring programs. Ecology 88, 49-55 (2007).

45 van de Pol, M. \& Wright, J. A simple method for distinguishing within- versus betweensubject effects using mixed models. Animal Behaviour 77, 753-758 (2009).

46 de Villemereuil, P., Wells, J., Edwards, R. \& Blomberg, S. Bayesian models for comparative analysis integrating phylogenetic uncertainty. BMC Evolutionary Biology 12, $102(2012)$.

47 Abadi, F. et al. Importance of accounting for phylogenetic dependence in multi-species mark-recapture studies. Ecological Modelling 273, 236-241 (2014).

48 Pagel, M. Inferring the historical patterns of biological evolution. Nature 401, 877-884 (1999).

49 Freckleton, R. P., Harvey, P. H. \& Pagel, M. Phylogenetic analysis and comparative data: A test and review of evidence. American Naturalist 160, 712-726 (2002). 
58050 Donoghue, M. J. \& Ackerly, D. D. Phylogenetic uncertainties and sensitivity analyses in

581 comparative biology. Philosophical Transactions of the Royal Society of London B:

582 Biological Sciences 351, 1241-1249 (1996).

58351 Jetz, W., Thomas, G. H., Joy, J. B., Hartmann, K. \& Mooers, A. O. The global diversity

$584 \quad$ of birds in space and time. Nature 491, 444-448 (2012).

58552 Spiegelhalter, D., Thomas, A., Best, N. \& Lunn, D. OpenBUGS user manual version $586 \quad 3.2 .3 .(2014)$.

58753 Sturtz, S., Ligges, U. \& Gelman, A. R2WinBUGS: a package for running WinBUGS 588 from R. Journal of Statistical Software 12, 1-16 (2005).

58954 BirdLife International. The BirdLife checklist of the birds of the world: Version 7 $590 \quad$ (Downloaded from

591 http://www.birdlife.org/datazone/userfiles/file/Species/Taxonomy/BirdLife_Checklist_V 592 ersion_70.zip. 2014).

59355 Paradis, E., Claude, J. \& Strimmer, K. APE: analyses of phylogenetics and evolution in R $594 \quad$ language. Bioinformatics 20, 289-290 (2004).

59556 Dowle, M. \& Srinivasan, A. data.table: extension of 'data.frame'. R package version $596 \quad 1.10 .4-3 .(2017)$.

59757 Wickham, H., Francois, R., Henry, L. \& Muller, K. dplyr: a grammar of data $598 \quad$ manipulation. $R$ package version 0.7.4. (2017).

59958 Wickham, H. ggplot2: elegant graphics for data analysis. (Springer-Verlag New York, 600 2016).

60159 Auguie, B. gridExtra: miscellaneous functions for "grid" graphics. R package version 2.3. 602 (2017).

60360 Brownrigg, R. mapdata: extra map databases. R package version 2.3.0. (2018). 
60461 Wickham, H. The split-apply-combine strategy for data analysis. Journal of Statistical $605 \quad$ Software 40, 1-29 (2011).

60662 Urbanek, S. png: read and write PNG images. R package version 0.1-7. (2013).

60763 Neuwirth, E. RColorBrewer: ColorBrewer Palettes. R package version 1.1-2. (2014).

60864 Bivand, R., Keitt, T. \& Rowlingson, B. rgdal: bindings for the geospatial data

$609 \quad$ abstraction library. R package version 1.2-8. (2017).

61065 Hijmans, R. J. raster: geographic data analysis and modeling. R package version 2.6-7.

$611 \quad$ (2017).

61266 Garnier, S. viridis: default color maps from 'matplotlib'. R package version 0.5.1. (2018).

\section{Acknowledgements}

615 We thank the coordinators, thousands of volunteer counters and funders of the International

616 Waterbird Census and Christmas Bird Count. T.A. was supported by the Grantham

617 Foundation for the Protection of the Environment, the Kenneth Miller Trust and the

618 Australian Research Council Future Fellowship (FT180100354). T.S. was funded by a Royal

619 Society Wolfson Merit Award (WM170050) and by the National Research, Development and

620 Innovation Office of Hungary (ÉLVONAL KKP-126949, K-116310). H.S.W. was supported

621 by the Cambridge Trust Cambridge-Australia Poynton Scholarship and the Cambridge

622 Department of Zoology JS Gardiner Fellowship. W.J.S. is supported by Arcadia and The

623 David and Claudia Harding Foundation. This work is also funded by EU Horizon 2020 BACI

624 project (Grant Agreement 640176), Ministry of the Environment of Japan, Environment

625 Canada, AEWA Secretariat, EU LIFE+ NGO Operational Grant, MAVA Foundation, Swiss

626 Federal Office for Environment and Nature, French Ministry of Environment and Sustainable

627 Development, UK Department of Food and Rural Affairs, Norwegian Nature Directorate, 
628 Dutch Ministry of Economics, Agriculture and Innovation, DOB Ecology and Wetlands

629 International members. Thanks to M. Amano for all the support.

\section{Author contributions}

631 T.A. designed the study. T.A., T.S., H.S.W., B.S., S.N., T.M., T.L., D.B. and N.L.M.

632 collected and prepared data for the analyses. T.A. analysed the data and wrote the paper. All

633 authors discussed the results and commented on the manuscript at all stages.

\section{Competing interests}

635 The authors declare no competing interests.

\section{Additional information}

637 Supplementary information is available for this paper.

638 Correspondence and requests for materials should be addressed to T.A.

639 (t.amano@uq.edu.au) 


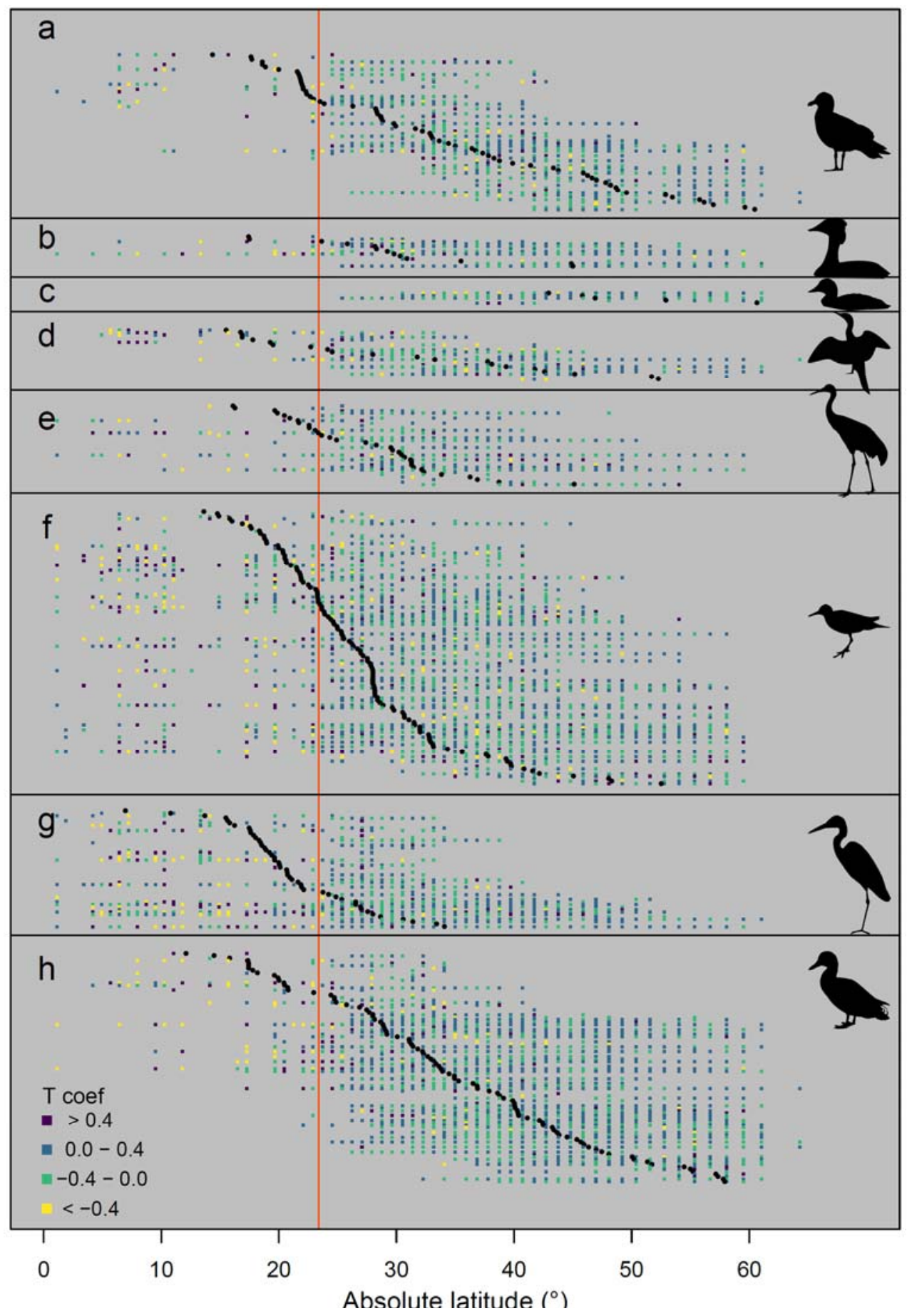

Fig. 1. Latitudinal distribution of abundance responses to changes in temperature (the rate of abundance changes with increasing temperature) for each species. Each horizontal row of squares shows the absolute latitudes of $1^{\circ} \times 1^{\circ}$ grid cells with estimates for each of the 390 species in (a) coursers, gulls, terns and auks, (b) grebes and flamingos, (c) loons and petrels, (d) pelicans, boobies and cormorants, (e) rails and cranes, (f) shorebirds, (g) storks, ibises and herons, and (h) waterfowl (see Methods for the definition of each species group). Black circles indicate the latitudinal range mid-point (i.e., median absolute latitude of geographical range) of each species. The area on the left of the red vertical line (absolute latitude $<23.4^{\circ}$ ) represents the tropical region. 

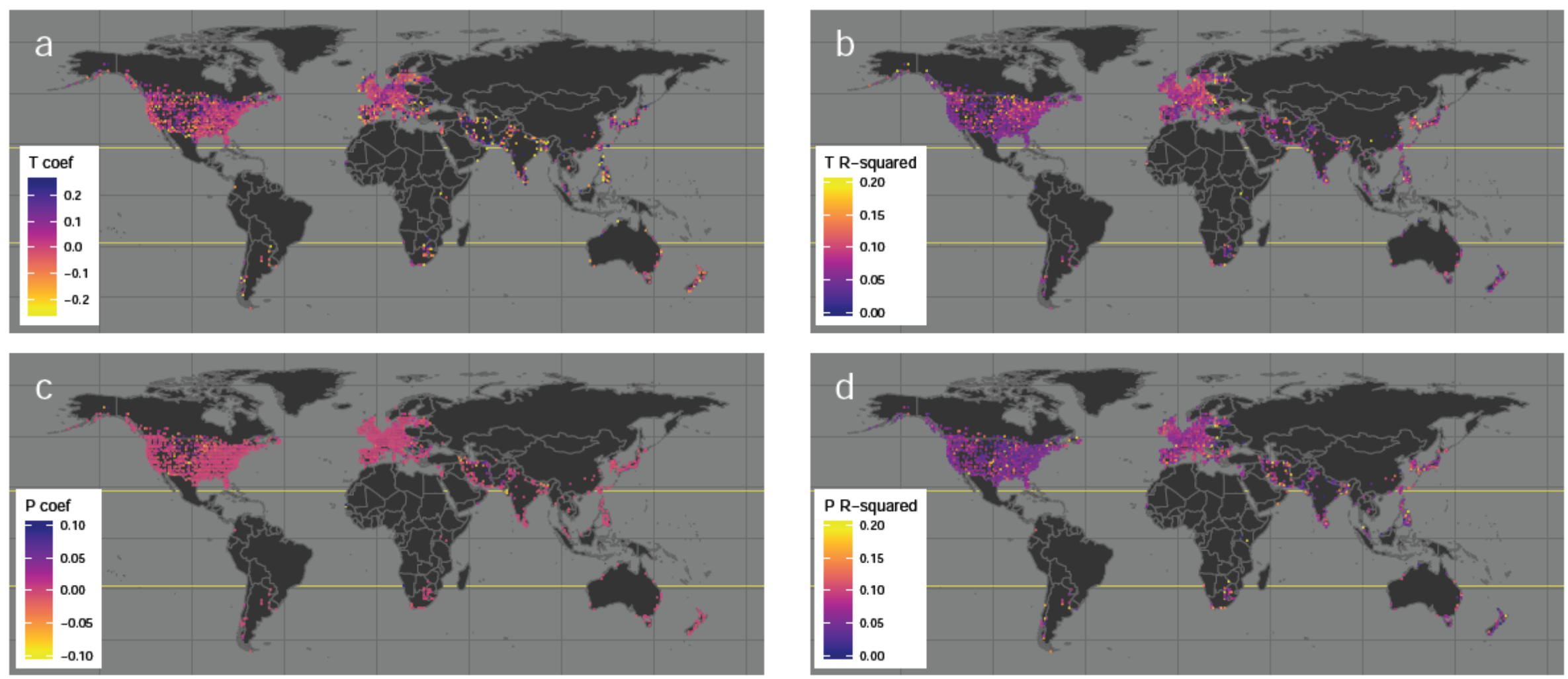

Fig. 2. Mean abundance responses across 390 waterbird species to changes in temperature and precipitation in each $1^{\circ} \times 1^{\circ}$ grid cell. The rate of abundance changes with increasing temperature (a), the independent capacity of temperature in explaining abundance changes (b), the rate region between the yellow solid lines is the tropics. 


\section{Among species}
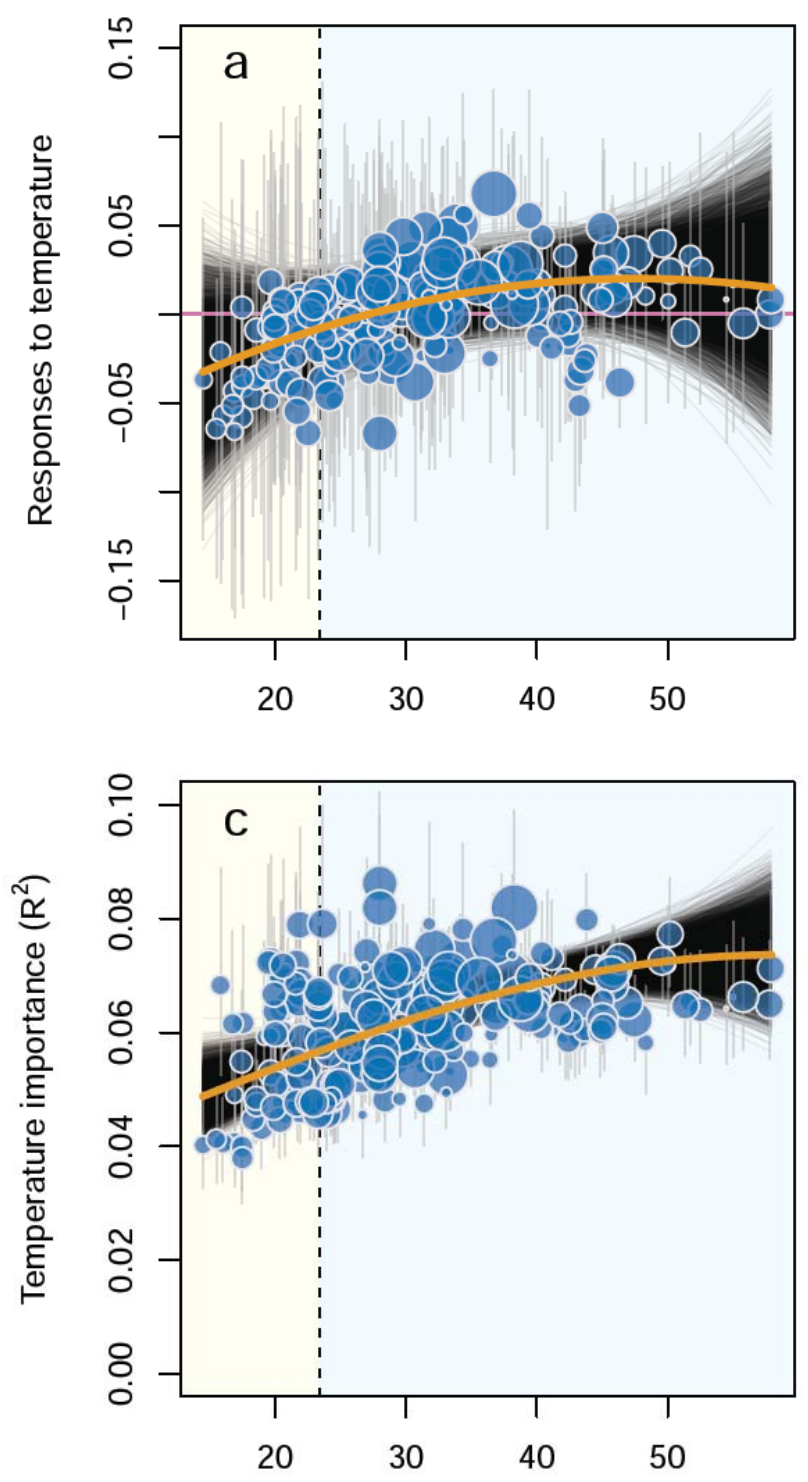

Within species
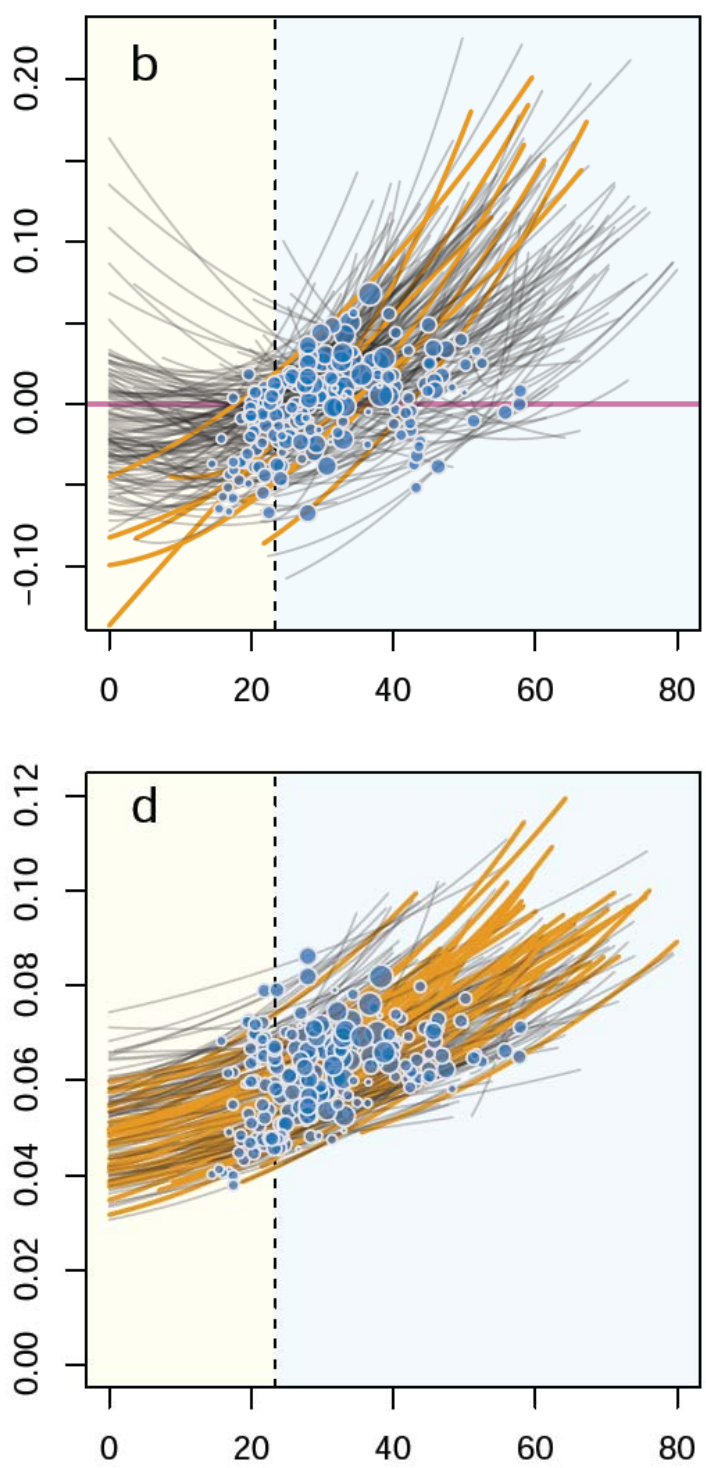

Absolute latitude $\left({ }^{\circ}\right)$

Fig. 3. Latitudinal patterns in waterbird abundance responses to temperature increases.

The rate of abundance changes with increasing temperature at each species' range mid-points (a) and within each species (b), the independent capacity of temperature in explaining abundance changes at each species' range mid-points (c) and within each species (d). In (a) and (c) orange lines represent the among-species latitudinal patterns based on posterior median coefficients with black lines showing patterns based on all posterior samples. Blue circles indicate the estimated responses at each species' range mid-points (also shown in (b) and (d)) with grey vertical lines showing $95 \%$ credible intervals; circle size, the absolute latitudinal range size. In (b) and (d) regression lines show the estimated within-species latitudinal patterns for each species based on posterior median coefficients; orange lines represent significant patterns. The yellow area (absolute latitude $<23.4^{\circ}$ ) represents the tropical region. 


\section{Among species}
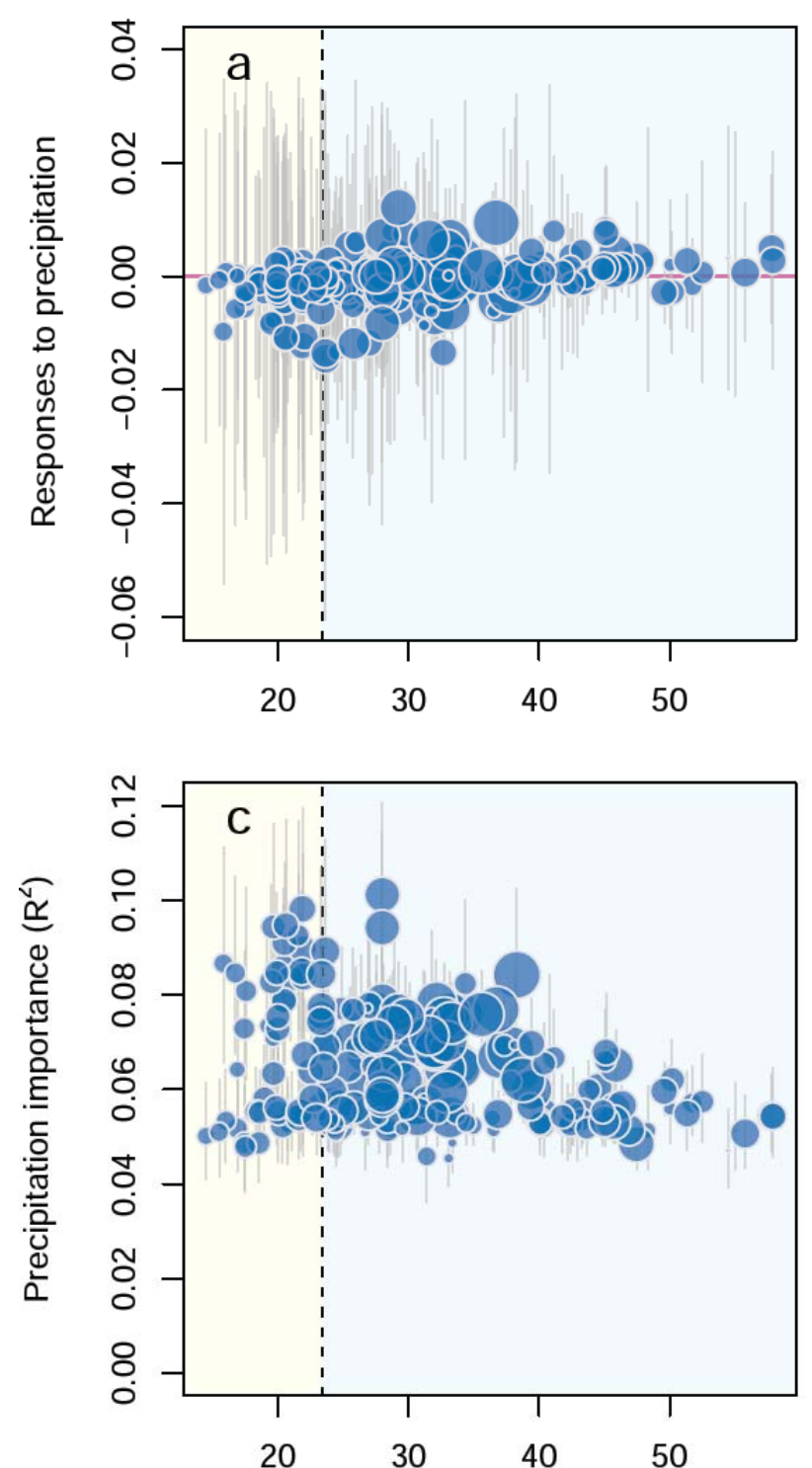
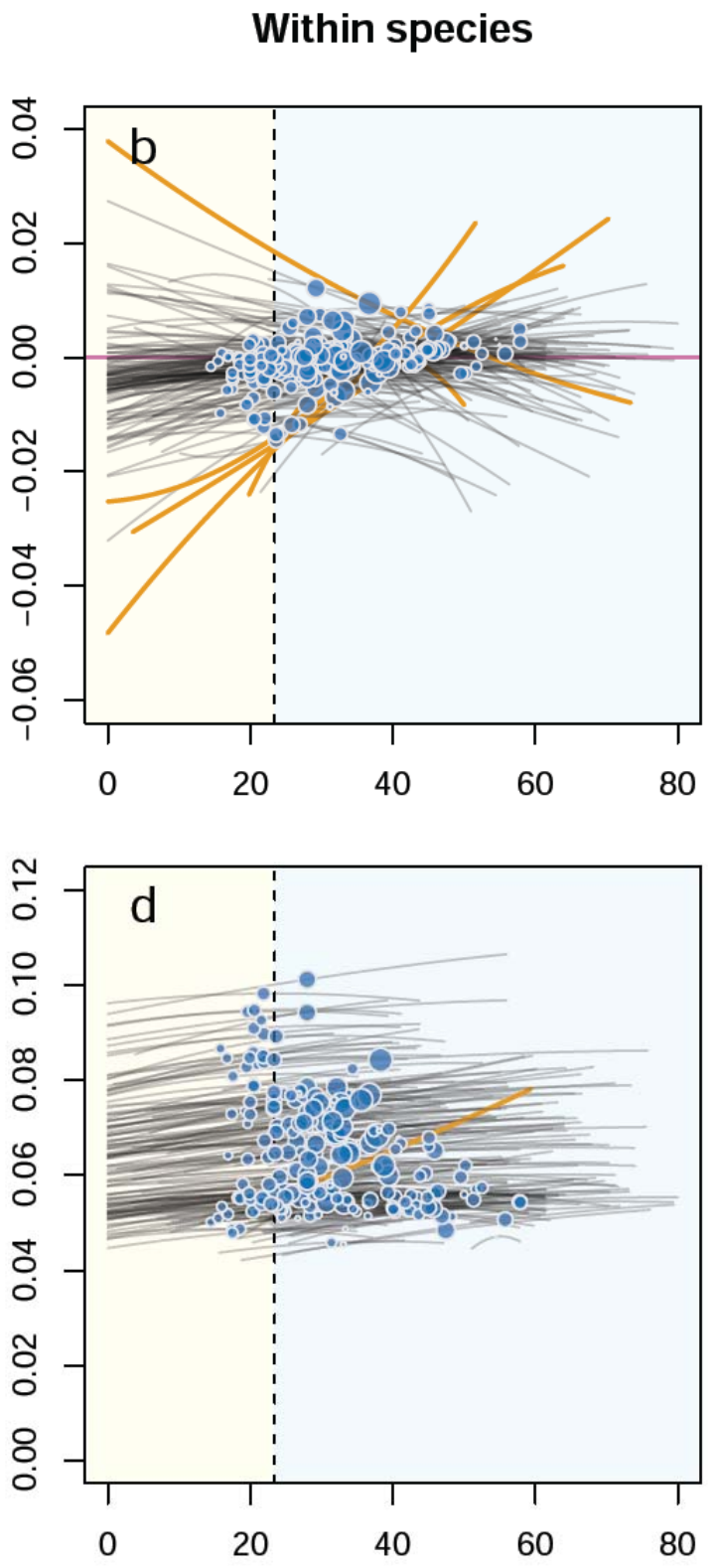

Absolute latitude $\left({ }^{\circ}\right)$

Fig. 4. Latitudinal patterns in waterbird abundance responses to precipitation increases.

The rate of abundance changes with increasing precipitation for each species' range midpoints (a) and within each species (b), the independent capacity of precipitation in explaining abundance changes at each species' range mid-points (c) and within each species (d). In (a) and (c) orange lines represent the among-species latitudinal patterns based on posterior median coefficients with black lines showing patterns based on all posterior samples. Blue circles indicate the estimated responses at each species' range mid-points (also shown in (b) and (d)) with grey vertical lines showing 95\% credible intervals; circle size, the absolute latitudinal range size. In (b) and (d) regression lines show the estimated within-species latitudinal patterns for each species based on posterior median coefficients; orange lines represent significant patterns. The yellow area (absolute latitude $<23.4^{\circ}$ ) represents the tropical region. 